\title{
A multichannel relay MAC protocol for IEEE 802.11 wireless LANs
}

\author{
Dong-Wook Kim ${ }^{1}$, Wan-Seon $\mathrm{Lim}^{2}$ and Young-Joo Suh ${ }^{3, *, \dagger}$ \\ ${ }^{1}$ Smart Grid Security Research Department, the Attached Institute of ETRI, Daejeon, Korea \\ ${ }^{2}$ Real-Time Computing Laboratory, Department of Electrical Engineering and Computer Science, The University of \\ Michigan, Ann Arbor, MI 48109-2121, USA \\ ${ }^{3}$ Division of IT Convergence Engineering (ITCE) and Department of Computer Science Engineering (CSE), POSTECH, \\ Pohang, Korea
}

\begin{abstract}
SUMMARY
One of the challenging issues in wireless LANs (WLANs) is improving the network throughput. One of the possible solutions for the issue is maximizing the number of concurrent transmissions. Although some protocols have been proposed to exploit transmission concurrency in WLANs, their performance depends on the degree of the interference among links. Also, it is hard to obtain interference information because of their dynamics. In this paper, we propose an enhanced medium access control (MAC) protocol for WLANs, named multichannel relay MAC (MRMAC), which is able to transmit multiple frames simultaneously without considering interference. To enable concurrent transmissions, MRMAC adopts the concept of frame relaying. Furthermore, MRMAC utilizes several nonoverlapping channels to eliminate interferences. Through extensive simulations, we found that MRMAC shows better performance than existing well-known MAC protocols. Copyright (C) 2013 John Wiley \& Sons, Ltd.
\end{abstract}

Received 30 August 2011; Revised 19 November 2012; Accepted 18 January 2013

KEY WORDS: concurrent transmission; multichannel; relay mechanism; medium access control; 802.11 wireless LANs

\section{INTRODUCTION}

IEEE 802.11-based wireless LANs (WLANs) [1] have become popular these days thanks to their low cost and easy access. Recently, most mobile devices such as laptops and mobile phones are equipped with WLAN interfaces. Because of the explosive use of WLAN devices, providing high network throughput under limited bandwidth becomes one of the most challenging issues.

Maximizing the number of concurrent transmissions can improve the network throughput. One way to increase transmission concurrency is to exploit spatial reuse. There have been extensive efforts [2-9] to increase the degree of concurrency under a single channel by exploiting spatial reuse via tuning transmission power, carrier sensing threshold, and data rate. However, if multiple links are close to each other such that they can interfere with each other, it is difficult to achieve concurrency. Another way to increase the transmission concurrency is to utilize multiple channels where IEEE 802.11a and IEEE 802.11b support 12 and 3 nonoverlapping channels, respectively. There is no interference among links that use different nonoverlapping channels. Several multichannel medium access control (MAC) protocols [10-12] have been designed to exploit nonoverlapping channels efficiently in multihop wireless networks. Because these protocols provide interference-free concurrent transmissions regardless of nodes' proximity, the network throughput in multichannel environments can be improved.

*Correspondence to: Young-Joo Suh, Division of ITCE and Department of CSE, POSTECH, Pohang, Korea.

†E-mail: yjsuh@postech.ac.kr 
These protocols $[2-8,10-12]$ work well in multihop wireless networks where nodes can directly communicate with each other. However, because two or more transmissions are not activated simultaneously in an infrastructure WLAN where all communications take place via access point (AP), it is difficult to adopt those protocols in an infrastructure WLAN. Recently, Hu et al. $[13,14]$ have proposed new protocols for enabling concurrent transmissions in WLANs by adopting a frame-relaying mechanism. A frame-relaying mechanism in [15-18] is used to improve network throughput by exploiting faster two-hop transmissions via relay nodes, whereas it is used in $[13,14]$ to make multiple links so that simultaneous transmissions of multiple links are possible even in the infrastructure WLAN. However, these protocols have a limitation of the possibility of transmission concurrency being dependent on the degree of interference among links.

In this paper, we propose a new MAC protocol for an infrastructure WLAN, called multichannel relay MAC (MRMAC), which is able to transmit multiple frames simultaneously without interference by utilizing several nonoverlapping channels in IEEE 802.11 WLANs. We design a decision algorithm for determining a set of frames to be transmitted and relay nodes participating in the transmissions and for allocating channels to be used for transmissions. Also, we propose a medium access operation of MRMAC for the concurrent frame transmissions. We performed extensive simulations based on ns-2 [19] to evaluate the performance of MRMAC. Our evaluation results demonstrate that MRMAC shows better throughput and delay performance than existing protocols.

The remainder of this paper is organized as follows. In Section 2, we describe background and related work. In Section 3, we present the motivation of this work. Then, the detailed operation of MRMAC is explained in Section 4. We evaluate the performance of MRMAC through extensive simulations in Section 5, and then we close the paper with concluding remarks in Section 6.

\section{BACKGROUND AND RELATED WORK}

Recently, the concept of using relay nodes to improve the throughput of wireless networks has received much attention in the literature [15-18,20-23]. The key motivation of the studies is that communications through relay nodes located between a transmitter and receiver can provide higher link quality than direct link. Several relay MAC protocols [15-18] exploiting the feature have been proposed. Because multihop relaying at the MAC layer may support higher data rates than a singlehop transmission, multihop transmissions via relay can reduce data transmission time compared with direct transmission and thus improve the network throughput. Generally, if the following relay condition is satisfied, frame relaying can be used to improve network throughput.

$$
\frac{1}{R_{\mathrm{S}, \mathrm{R}}}+\frac{1}{R_{\mathrm{R}, \mathrm{D}}}<\frac{1}{R_{\mathrm{S}, \mathrm{D}}}
$$

where $R_{\mathrm{S}, \mathrm{R}}, R_{\mathrm{R}, \mathrm{D}}$, and $R_{\mathrm{S}, \mathrm{D}}$ denote the transmission rates between a transmitter and relay node, a relay node and receiver, and a transmitter and receiver, respectively.

Figure 1 shows DATA (data frame) transmission procedure of a relay-aided media access (RAMA) protocol [16]. In RAMA, when the relay condition is satisfied for a link pair between transmitter and receiver, a relay node broadcasts an invitation frame to notify a node that it is a candidate relay node. When a transmitter has DATA to be transmitted to a receiver, it transmits the frame to the receiver via the relay node. For example, as shown in Figure 1, after request to send (RTS) and clear to send (CTS) are exchanged between a transmitter and receiver, the transmitter sends DATA to relay1. Upon receiving DATA from the transmitter, relay1 forwards the frame to the receiver without channel contention. When the receiver receives DATA from relay 1, it transmits an acknowledgement (ACK) to the transmitter as a response. Because two-hop relaying transmissions via a relay node take less time than a direct transmission, frame-relaying approaches can improve throughput.

Many research efforts have concentrated on increasing transmission concurrency to improve network throughput in 802.11-based wireless networks. There have been studies for improving spatial reuse under a single channel by adjusting carrier sensing threshold [2,3], transmit power [4-6], and so on [7-9]. For example, the carrier sensing threshold adjustment in [3] has designed an 


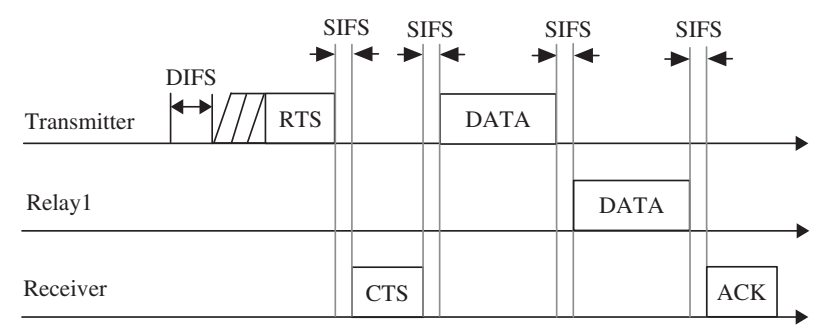

Figure 1. DATA transmission procedure in relay-aided media access [16]. DIFS, distributed coordination function interframe space; SIFS, short interframe space.

algorithm that can find the optimal carrier sense threshold on the basis of the signal-to-interference and noise ratio threshold for a certain rate, and thereby spatial reusability can be improved. In [5], on the basis of the maximum tolerable interference information that is advertised by the receiver, the transmitter selects a proper transmit power that does not interfere with ongoing transmissions. In [7-9], the transmit power, data rate, or carrier sensing threshold are jointly tuned to improve network capacity.

In [10-12], several multichannel-based MAC protocols under a single transceiver have been proposed for multihop wireless networks. The basic idea is to eliminate interference among close links by utilizing the multichannel capability of IEEE 802.11. For example, IEEE $802.11 \mathrm{~b}$ supports three nonoverlapping channels such as channels 1, 6, and 11. The multichannel MAC protocol [10] attempts to improve network capacity by allowing simultaneous transmissions among multiple links under nonoverlapping channels. In multichannel MAC, a pair of nodes periodically switches to the common control channel for channel negotiation and reservation during the control phase, and then it switches to the reserved channel to transmit data frame during the data phase. Improving spatial reuse by adjusting tunable parameters and exploiting multichannel capability works well in ad hoc wireless networks where all nodes can communicate with each other without infrastructures such as AP. However, these protocols are hard to adopt in infrastructure-based wireless networks.

There also have been research efforts utilizing a frame-relaying mechanism to allow concurrent transmissions $[13,14]$ under the single channel. Hu et al. [14] have proposed the sender initiating concurrent cooperative MAC (SI-CCMAC) protocol that uses a two-hop relaying mechanism and enables downlink concurrent transmissions through coordination. In SI-CCMAC, AP decides a set of frames to be transmitted that consist of several receivers and different relay nodes for each selected receiver where they cause low interference to each others. Then, AP transmits each data frame to each relay node back to back when it seizes the medium. All selected relay nodes transmit the data frames to its corresponding receivers at the same time without contention after they received data frames from AP.

\section{MOTIVATION}

In this section, to explain the advantage of exploiting concurrent transmissions compared with both direct transmissions and relaying transmissions, we will show the achievable throughput of those approaches.

Suppose that AP has data frames of $L$ bytes to transmit to both nodes D1 and D2 and the transmission rate is $2 \mathrm{Mb} / \mathrm{s}$. There are two relay nodes (i.e., nodes R1 and R2), and the transmission rates of $\mathrm{AP} \rightarrow \mathrm{R} 1, \mathrm{R} 1 \rightarrow \mathrm{D} 1, \mathrm{AP} \rightarrow \mathrm{R} 2$, and $\mathrm{R} 2 \rightarrow \mathrm{D} 2$ are $5.5,11,5.5$, and $11 \mathrm{Mb} / \mathrm{s}$, respectively, as shown in Figure 2. In the case of two continuous direct transmissions, we can expect the achievable throughput of $2 \mathrm{Mb} / \mathrm{s}$. When a frame-relaying mechanism is used in the scenario, the achievable throughput becomes $3.67 \mathrm{Mb} / \mathrm{s}$ (i.e., $2 L / 2(L / 5.5 M+L / 11 M)$ ). If two flows do not interfere with each other such as $\mathrm{R} 1 \rightarrow \mathrm{D} 1$ and $\mathrm{R} 2 \rightarrow \mathrm{D} 2$ in Figure 2, concurrent transmissions are possible as follows. AP transmits a data frame destined to node D1 to node R1, and then AP transmits a data frame destined to node $\mathrm{D} 2$ to node $\mathrm{R} 2$. Then, nodes $\mathrm{R} 1$ and $\mathrm{R} 2$ send the received frames to D1 and D2 simultaneously. Thus, achievable throughput can further be increased to $4.4 \mathrm{Mb} / \mathrm{s}$ 


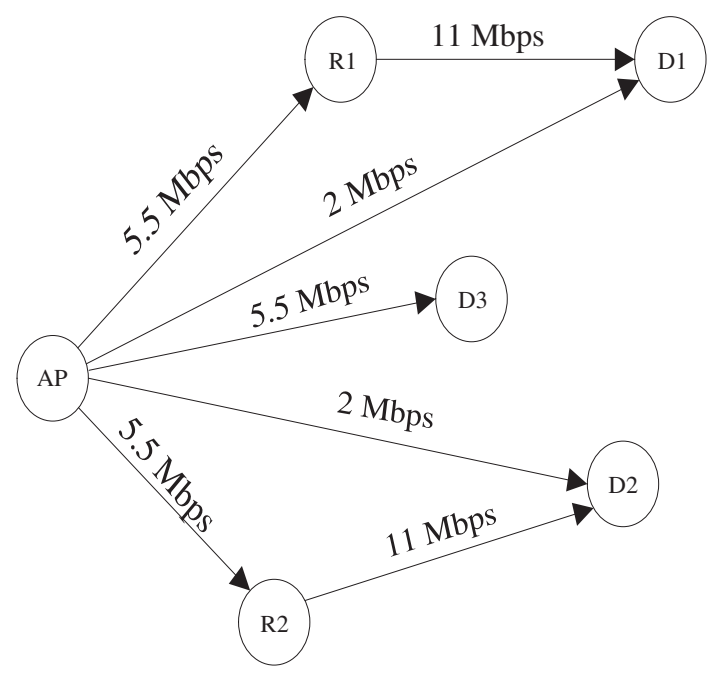

Figure 2. An example scenario to show the advantage of using concurrent transmission. AP, access point.

(i.e., $2 L /(L / 5.5 M+L / 5.5 M+L / 11 M))$ with the help of transmission concurrency. Although control overhead can decrease the actual throughput, we can still expect improved throughput, if the frame size is not too small $[13,14]$.

However, using concurrent transmissions in a single channel has several limitations as follows. First, AP should be aware of the interference information of all possible links in the network. It is possible for AP to know the interference map among all links in the network if each node notifies AP of its perceived interference level from all the other nodes. This may cause much control overhead. Second, the AP has difficulty maintaining the accurate interference level of each node experienced by the other nodes in mobile scenarios. Another limitation of the approach is the possibility of transmission concurrency being dependent on the degree of interference among nodes. As an example, if two links (links R1 $\rightarrow \mathrm{D} 1$ and $\mathrm{R} 2 \rightarrow \mathrm{D} 2$ in Figure 2) interfere with each other, both links cannot be activated simultaneously. To handle this, we propose MRMAC, which utilizes nonoverlapping channels to eliminate interference so that it allows multiple frames to be transmitted simultaneously without interference.

\section{MULTICHANNEL RELAY MEDIUM ACCESS CONTROL PROTOCOL}

In this section, we describe the operation of the proposed protocol called MRMAC, which exploits the frame relay mechanism and nonoverlapping channels to enable interference-free concurrent transmissions in a WLAN. First, we explain the system model of MRMAC. Next, we will describe the relay node notification procedures and the maintenance of the relay nodes at both AP and other nodes. Then, we will explain the decision algorithm performed in AP to prepare a set of frames to be transmitted at the channel access. After explaining the decision algorithm, we describe the medium access operation among AP, relay nodes, and receivers. Last, we explain the effect of channel switching delay and throughput analysis and discuss some issues considered for incorporating MRMAC into IEEE 802.11.

\subsection{System model}

First, we explain the system model in MRMAC. We assume that a single AP and several nodes exist in an infrastructure WLAN. Also, there is no energy constraint of node, node failure, and network partitioning. But we assume that frame loss may occur because of either collision by hidden node or channel error. And all nodes are working on the distributed coordination function (DCF)mode-based IEEE 802.11 and can overhear the ongoing frames even if they are encoded. AP takes responsibility for transmitting frames to the intended receivers, whereas the other nodes transmit 
their frames toward AP. The nodes except AP can act as relay nodes for some links. Last, AP can obtain the information about unused or rarely used channel by adjacent APs.

\subsection{Relay node notification and maintenance}

In MRMAC, nodes can act as relay nodes if some conditions are satisfied. If those nodes exist, they will perform relay node notification procedure periodically to notify AP about the information that it is a candidate relay node for some links. AP maintains the received information in a table, referred to as RelayTable, and uses it when executing the decision algorithm, explained in the next subsection.

Now, we explain how nodes can know that it can be a relay node. In 802.11-based wireless networks, nodes between a transmitter and receiver can overhear ongoing frames (e.g., DATA or ACK) between them. When a node overhears a data frame and if it can decode that frame, it can know the transmission rate between the transmitter and receiver from the physical layer convergence protocol (PLCP) header of a data frame (i.e., $R_{\mathrm{S}, \mathrm{D}}$ ) and the MAC addresses of both the transmitter and receiver from the MAC header of the frame. Also, the node can estimate the channel condition between the transmitter and itself by measuring the signal strength of the received data frame. From the signal strength, the node determines the supportable transmission rate between the transmitter and itself (i.e., $R_{\mathrm{S}, \mathrm{R}}$ ) similar to that in [24]. Specifically, a node compares the received signal strength with the threshold value, which can satisfy a required bit error rate for the each modulation scheme. By doing so, it can choose the most proper transmission rate for each node. When the intended receiver of a data frame decodes the data frame successfully, it will send ACK as a response after a short interframe space (SIFS). Thus, although ACK has no transmitter's MAC address field, the node overhearing both ACK and a data frame can infer the MAC address of the transmitter. From the received signal strength of ACK, the node overhearing ACK can choose the transmission rate between itself and the receiver (i.e., $R_{\mathrm{R}, \mathrm{D}}$ ). In addition, both RTS and CTS can be also used to measure channel conditions.

Once a node acquires $R_{\mathrm{S}, \mathrm{D}}, R_{\mathrm{S}, \mathrm{R}}$, and $R_{\mathrm{R}, \mathrm{D}}$, it determines whether it can be a relay node or not for data transmissions between the transmitter and receiver by checking the condition in Equation (1). If the condition is satisfied, it can act as a candidate relay node for the link pair between the transmitter and receiver. The candidate relay node stores the corresponding information in a table that has five fields: MAC addresses of the transmitter and receiver, $R_{\mathrm{S}, \mathrm{R}}, R_{\mathrm{R}, \mathrm{D}}$, and $R_{\mathrm{S}, \mathrm{D}}$. A candidate relay node prepares an advertisement message that contains its table information and forwards it to AP periodically.

When receiving the advertisement message, AP stores the corresponding information in the RelayTable, which has five fields for each entry: STA ID, RESTA ID, $R_{\mathrm{S}, \mathrm{R}}, R_{\mathrm{R}, \mathrm{D}}$, and $R_{\mathrm{S}, \mathrm{D}}$. STA ID and RESTA ID denote the MAC addresses of the receiver and relay nodes, respectively. The table has one or more entries for a STA ID, which means that one or more nodes can be candidate relay nodes for the receiver. AP maintains the latest RelayTable information to avoid abnormal operations. For this, whenever receiving advertisement messages from candidate relay nodes or receiving control frames (such as CTS and ACK) from some nodes, AP updates the entries associated with the received messages in its RelayTable. Also, for stale information to be dealt with, each entry in the table is maintained by a timer. If AP cannot receive any information for a certain entry until the expiration of the timer, it deletes the entry from the RelayTable.

Similar to the AP, nodes have staRelayTable, which is used to find relay nodes for faster transmission of their own uplink frames. When a node overhears an advertisement message from a certain node, it checks its MAC address in the message. If it is found, the node stores the associated information in its staRelayTable, which has four fields for each entry: RESTA ID, $R_{\mathrm{S}, \mathrm{R}}, R_{\mathrm{R}, \mathrm{D}}$, and $R_{\mathrm{S}, \mathrm{D}}$. Nodes maintain their table with the latest information by a timer similar to APs.

\subsection{Decision algorithm}

In MRMAC, before the channel access, AP executes the decision algorithm consisting of both the frame selection and the channel assignment, which selects a set of frames and allocates channels to those frames, respectively. 


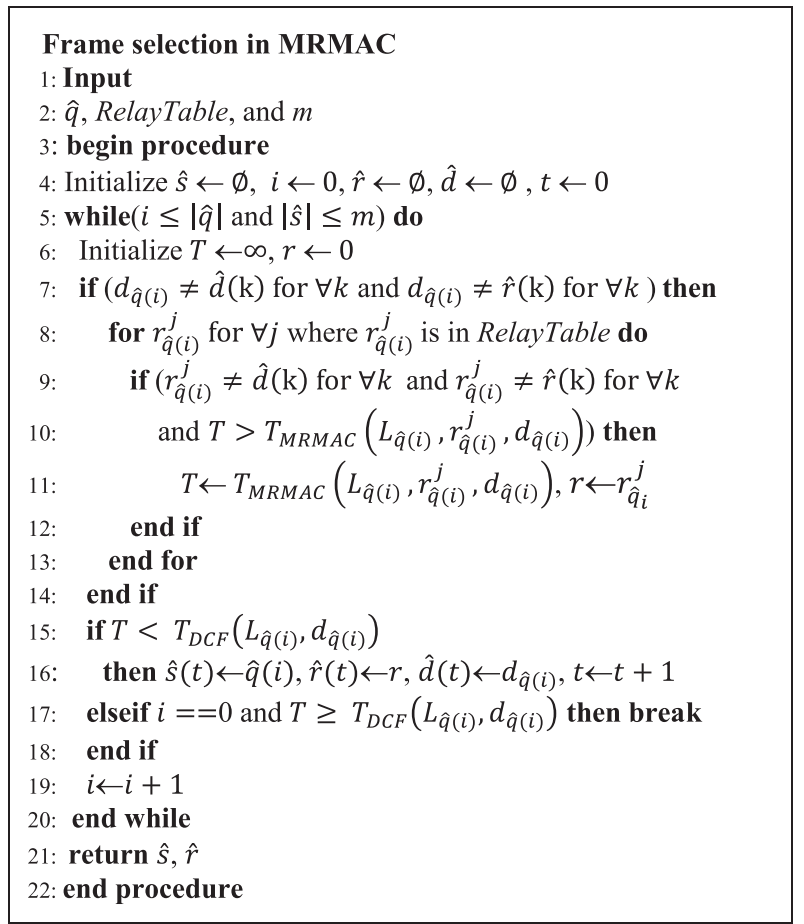

Figure 3. Frame selection part in decision algorithm. DCF, distributed coordination function; MRMAC, multichannel relay medium access control.

Table I. Notations used in multichannel relay medium access control.

\begin{tabular}{ll}
\hline Notations & \multicolumn{1}{c}{ Definitions } \\
\hline$m$ & The number of available nonoverlapping channels \\
$\hat{q}$ & A list of frames in the access point's queue \\
$\hat{s}$ & A list of selected frames to be transmitted in $\hat{q}$ \\
$\hat{d}$ & A list of the receiver address for each frame in $\hat{s}$ \\
$\hat{r}$ & A list of the selected relay node address for each frame in $\hat{s}$ \\
$|\hat{x}|$ & The number of elements in $\hat{x}(x \in\{q, s, d, r\})$ \\
$\hat{x}(i)$ & $i$ th element in $\hat{x}$ \\
$d_{\hat{q}(i)}$ & The receiver of frame $\hat{q}(i)$ \\
$r_{\hat{q}}^{j}(i)$ & A candidate relay node $j$ of frame $\hat{q}(i)$ \\
$L_{\hat{q}(i)}$ & The packet length of frame $\hat{q}(i)$ \\
\hline
\end{tabular}

First, we explain the frame selection algorithm where $m$ is the number of available nonoverlapping channels. The frame selection algorithm is used to determine the following: (i) a set of frames to be transmitted; and (ii) a set of nodes acting as a relay node for each selected frame. Figure 3 shows the details of frame selection, and Table I shows the notations used in the decision algorithm. For the while loop (steps 5-20), AP checks frames in the queue one by one until either no more frames exist in the queue or the number of selected frames is greater than $m$. We limit $|\hat{s}|$ to $m$ to guarantee interference-free transmissions among selected frames.

In each iteration, AP first checks whether the receiver for the $i$ th frame of the queue is the same as either the receiver or its relay node for each of the selected frames or not (step 7). The reason for the step is to make several disjoint links for enabling concurrent transmissions among them. If step 7 is satisfied, the AP examines its RelayTable by using the receiver of the $i$ th frame as the index to find a possible relay node (step 8). The candidate relay nodes should satisfy the condition that 
they are neither the receivers nor the relay nodes for the selected frames (step 9). Then, a node with minimum $T_{\text {MRMAC }}$ among candidate relay nodes is selected as a relay node for the frame (step 11). $T_{\text {MRMAC }}(L, R, D)$ means the time required for transmitting a frame of $L$ bytes to receiver $D$ via relay node $R$, which is expressed as

$$
T_{\mathrm{MRMAC}}(L, R, D)=T\left(L, R_{\mathrm{AP}, \mathrm{R}}\right)+T\left(L, R_{\mathrm{R}, \mathrm{D}}\right)+3 t \operatorname{SIFS}+2 T_{\mathrm{ACK}}
$$

where $T_{\mathrm{ACK}}$ is the transmission time for $\mathrm{ACK}, T\left(L, R_{\mathrm{AP}, \mathrm{R}}\right)$ is the transmission time of a data frame with $R_{\mathrm{AP}, \mathrm{R}}$ (the transmission rate between $\mathrm{AP}$ and relay node $\left.R\right), T\left(L, R_{\mathrm{R}, \mathrm{D}}\right)$ is the transmission time of a data frame with $R_{\mathrm{R}, \mathrm{D}}$ (the transmission rate between relay node $R$ and receiver $D$ ). Then, AP compares the minimum $T_{\mathrm{MRMAC}}(L, R, D)$ with $T_{\mathrm{DCF}}(L, D)$. $T_{\mathrm{DCF}}(L, D)$ means the time required for transmitting a frame of $L$ bytes to receiver $D$ directly and is expressed as

$$
T_{\mathrm{DCF}}(L, D)=T\left(L, R_{\mathrm{AP}, \mathrm{D}}\right)+t S I F S+T_{\mathrm{ACK}}
$$

where $T\left(L, R_{\mathrm{AP}, \mathrm{D}}\right)$ is the transmission time of a data frame with $R_{\mathrm{AP}, \mathrm{D}}$ (the transmission rate between AP and receiver $D$ ). If $T_{\mathrm{MRMAC}}$ is less than $T_{\mathrm{DCF}}$, the frame is inserted into $\hat{s}$, and the receiver and its selected relay node are also inserted into $\hat{d}$ and $\hat{r}$, respectively (steps 15 and 16). Then, if $|\hat{s}|$ is smaller than $m$, AP goes to step 6 and executes the algorithm again with the next frame in the $\hat{q}$.

If there is no candidate relay node that satisfies steps 9 and 10 or if the minimum $T_{\text {MRMAC }}$ is larger than $T_{\mathrm{DCF}}$, the next step is as follows. If the frame is the first frame in the queue (i.e., $i$ equals 0 in step 17), AP stops the frame selection part. Otherwise, AP continues the frame selection algorithm for the next frame of $\hat{q}$ (step 6). As results of the frame selection algorithm, $\hat{s}$ and $\hat{r}$ can be obtained by AP, which will be used in the medium access operation. To reduce the processing time, AP only keeps a pointer of each selected frame in $\hat{s}$ instead of a copy for each one.

After executing the frame selection algorithm, AP performs the channel assignment algorithm, which allocates a different channel to each selected frame. The allocated channel is used to transmit a data frame between the receiver of the frame and its relay node. There are two types of channels in MRMAC: a primary channel and a secondary channel. The primary channel is a main channel used for transmissions between AP and its associated nodes in a WLAN. The secondary channel is any channel except the primary channel, and AP exploits unused or rarely used channels as secondary channels.

When the number of selected frames (i.e., $|\hat{s}|$ ) is 1 , AP allocates the frame to the primary channel. Otherwise (i.e., $|\hat{s}| \geqslant 2$ ), AP allocates a different nonoverlapping channel to each of the selected frames as follows. AP allocates the primary channel to a frame that has the maximum $T\left(L, R_{\mathrm{R}, \mathrm{D}}\right)$ value in $\hat{s}$, whereas the other channels (i.e., secondary channel) are assigned randomly to the remaining frames in $\hat{s}$. Then, AP sorts both $\hat{s}$ and $\hat{r}$ such that the frame using the primary channel is served last, whereas the others are served randomly. The reason why the frame with the maximum $T\left(L, R_{\mathrm{R}, \mathrm{D}}\right)$ value is served last is to allow nodes using a secondary channel to participate in the next channel contention in time. Suppose that the data transmission under the primary channel is terminated but the data transmission under a secondary channel is not terminated yet. In this case, the nodes using the secondary channel cannot participate in the next channel contention in time. To avoid such problem, AP allocates a frame with the maximum $T\left(L, R_{\mathrm{R}, \mathrm{D}}\right)$ value to the primary channel where the frame is transmitted at last.

In a decision algorithm, if the first frame in AP's queue is able to be transmitted via relay nodes, the frame selection part could choose multiple frames to be relayed regardless of their queue positions. Thus, some relay frames arriving in the AP's queue later than some direct transmission frames might be served in advance. However, because AP always transmits the first frame of the queue in a channel access, the serving opportunity to be transmitted is the same between those frames. The computational complexity of the decision algorithm depending on $|\hat{q}|, N$, and $m$ is represented as $\mathrm{O}\left(|\hat{q}| \cdot N \cdot m^{2}+|\hat{s}| \cdot \log |\hat{s}|\right)=\mathrm{O}\left(|\hat{q}| \cdot N \cdot m^{2}\right)$, where $N$ is the number of elements in the RelayTable. Because the complexity is relatively small, it is possible for AP to finish the execution of the decision algorithm before the backoff expiration. However, if AP does not complete the execution of the algorithm by the end of backoff time, it will do the backoff procedure again. 


\begin{tabular}{|c|c|c|c|c|c|c|c|}
\hline Bytes: 2 & 2 & 6 & 6 & 1 & 13 & 13 & 4 \\
\hline $\begin{array}{c}\text { Frame } \\
\text { Control }\end{array}$ & Duration & $\begin{array}{c}\text { Bcast } \\
\text { address }\end{array}$ & $\begin{array}{c}\mathrm{AP} \\
\text { address }\end{array}$ & $\begin{array}{c}\text { TX } \\
\text { num (j) }\end{array}$ & $\begin{array}{c}\text { TX } \\
\text { list1 } \\
\end{array}$ & $\begin{array}{l}\text { TX } \\
\text { list j }\end{array}$ & FCS \\
\hline & & & & $\begin{array}{c}\text { Receiver } \\
\text { address }\end{array}$ & $\begin{array}{c}\text { Relay node } \\
\text { address }\end{array}$ & $\begin{array}{l}\text { Channel } \\
\text { number }\end{array}$ & \\
\hline
\end{tabular}

Figure 4. Frame structure of group request to send. AP, access point; FCS, frame check sequence; TX, transmission.

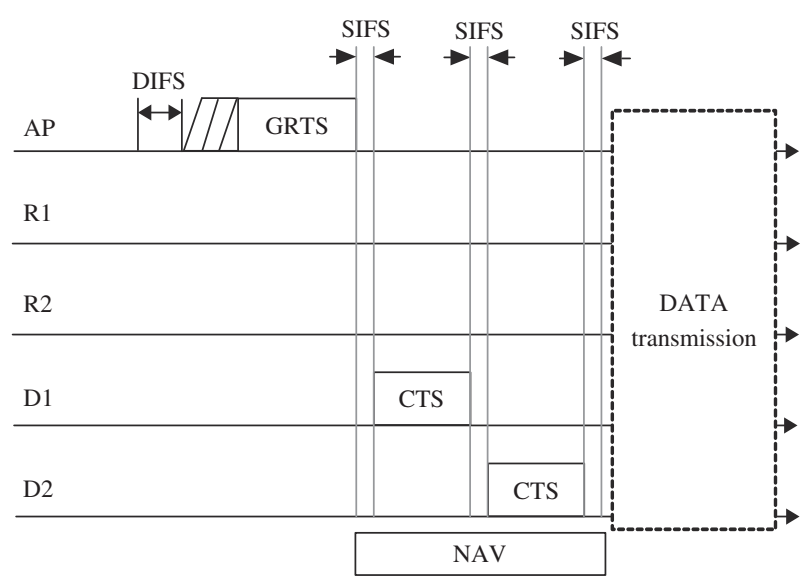

Figure 5. The timing diagram of group request-to-send (GRTS) and clear-to-send (CTS) transmission in multichannel relay medium access control. AP, access point; DIFS, distributed coordination function interframe space; NAV, network allocation vector; SIFS, short interframe space.

\subsection{Medium access operation}

Now, we describe the medium access operation of MRMAC. From the results of the decision algorithm, AP determines which transmission mode is used for data transmission among the DCF mode (i.e., $|\hat{s}|=0$ ), single-frame relay mode (i.e., $|\hat{s}|=1$ ), and multiple-frame relay mode (i.e., $|\hat{s}| \geqslant 2$ ).

In both single-frame and multiple-frame relay modes, AP first makes a group RTS (GRTS) frame whose frame format is shown in Figure 4, where the duration field means the required time for the reception of CTSs from all intended receivers, the TXnum field means the number of selected frames to be transmitted (i.e., $|\hat{s}|$ ), and the TXlist field consists of a receiver address, its relay node address, and a channel number assigned to the frame. The duration field is set to TXnum $\times T_{\mathrm{CTS}}+($ TXnum +1$) \times t$ SIFS, where $T_{\mathrm{CTS}}$ is the transmission time of CTS and tSIFS is the time length of SIFS. We can obtain the information filled in the TXnum field and several TXilst fields by the decision algorithm.

Figure 5 shows an example timing diagram of MRMAC where nodes exchange GRTS and two CTSs. In the example, the TXnum field in GRTS is set to 2. The receiver address, relay address, and channel number in TXlist1 are set to D1, R1, and a secondary channel (e.g., channel 6), respectively, whereas those in TXlist 2 are set to D2, R2, and the primary channel (e.g., channel 1), respectively. AP broadcasts GRTS after channel contention. From GRTS, each node knows whether it is an intended receiver or not and identifies its relay node (e.g., R1 for D1 and R2 for D2 in Figure 5). Also, they obtain the allocated channel number used for data transmissions. Then, the intended receivers send CTSs as a response. To avoid collisions, CTSs are transmitted one by one according to the order in the TXlist. In other words, before transmitting CTS, the receiver of the $i$ th TXlist waits for a time of $(i-1) \times T_{\mathrm{CTS}}+i \times t$ SIFS. In Figure 5, D1 should wait for $t$ SIFS, whereas D2 should wait for $T_{\mathrm{CTS}}+2 \times t$ SIFS before transmitting its CTS to AP. The CTS includes the information about $R_{\mathrm{AP}, \mathrm{D}}$ (the transmission rates between AP and a receiver $D$ ), where each receiver estimates the rate 


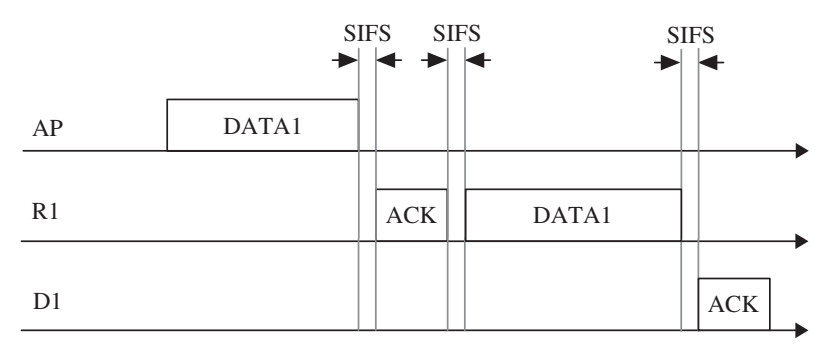

Figure 6. The timing diagram of DATA transmission in a single-frame relay mode. ACK, acknowledgement; AP, access point; SIFS, short interframe space.

from the channel condition of the received GRTS. Through overhearing CTS sent by a receiver, the selected relay node for the receiver estimates $R_{\mathrm{R}, \mathrm{D}}$ (the transmission rate between the receiver and the relay node). If one or more CTSs during the expected time are received, the AP starts data frame transmission procedures. It removes the corresponding element in $\hat{s}$ and $\hat{r}$ about receivers that did not send CTSs. If AP did not receive any CTSs from intended receivers, it removes all elements in $\hat{s}$ and $\hat{r}$ and stops the data frame transmission procedures.

Figure 6 illustrates the transmission procedure of the single-frame relay mode, which is similar to the operation of existing frame-relaying mechanisms [15-18]. After control frames are exchanged between AP and D1, AP gets DATA1 from $\hat{s}$ and transmits it to R1. The duration field is set to $t S I F S+T_{\mathrm{ACK}}$, which is the time required for the subsequent ACK reception. When $\mathrm{R} 1$ receives DATA1 successfully, it sends ACK as a response after $t$ SIFS. When AP receives ACK from R1, it deletes DATA1 in its queue (i.e., $\hat{q}$ ). Otherwise, AP will retransmit the frame. After transmitting ACK, R1 forwards DATA1 to the intended receiver (i.e., D1). Last, D1 responds to R1 with ACK.

Now, we explain the medium access operation in the multiple-frame relay mode when $|\hat{s}|$ is $m$ $(m \geqslant 2)$. After GRTS and $m$ CTSs are exchanged among AP and its selected receivers, AP obtains the first data frame from $\hat{s}$ and transmits it to the selected relay node for the frame. If AP receives ACK from the relay node that transmits ACK as a response, it removes the transmitted data frame in its queue (i.e., $\hat{q}$ ). Otherwise, AP will retransmit the frame at the next channel access. While waiting for the ACK reception, AP prepares for the transmission of the next frame in $\hat{s}$ in advance. Once the AP receives the ACK, it sends the next frame consecutively to its selected relay node after $t S I F S$, which is similar to the transmission opportunity operation in IEEE 802.11e [25]. AP continues back-to-back transmissions until the number of transmission trials is equal to $|\hat{s}|$.

Meanwhile, when a relay node receives a data frame from AP successfully, if the allocated channel is not the primary channel, it switches to the allocated channel right after transmitting ACK to AP. And the intended receiver switches to the channel for the communication with the relay node only if the relay node successfully receives a data frame from AP. When the channel is idle during $t D I F S$, which is the time length of the DCF interframe space (DIFS), the relay node starts exchanging DATA and ACK with the intended receiver. It removes the data frame when receiving ACK from the receiver as a response. Once frames are exchanged, they return to the primary channel and wait to participate in the next channel contention. Now, let us examine how the receiver checks whether the relay node receives a data frame successfully or not when determining channel switch. It is possible if the receiver overhears both a data frame and an ACK frame between AP and the relay node. However, the data frame is transmitted to the relay node with a rate higher than can be supported by the receiver, so the receiver cannot overhear the frame. Also, because ACK has no transmission field, although the receiver can overhear ACK, it cannot know whether the ACK frame is sent by its relay node or not. To deal with this problem, we modify ACK to include the MAC address of the transmitter.

As an example, we explain the transmission procedure of the multiple-frame relay mode when $|\hat{s}|$ is 2, which is shown in Figure 7. After GRTS and CTSs are exchanged among AP, D1, and D2 in Figure 5, AP extracts DATA1 from $\hat{s}$ and transmits it to R1. If AP receives ACK from R1 as a response, it removes the DATA1 in its queue. Then, AP obtains DATA2 from $\hat{s}$ and transmits it to 


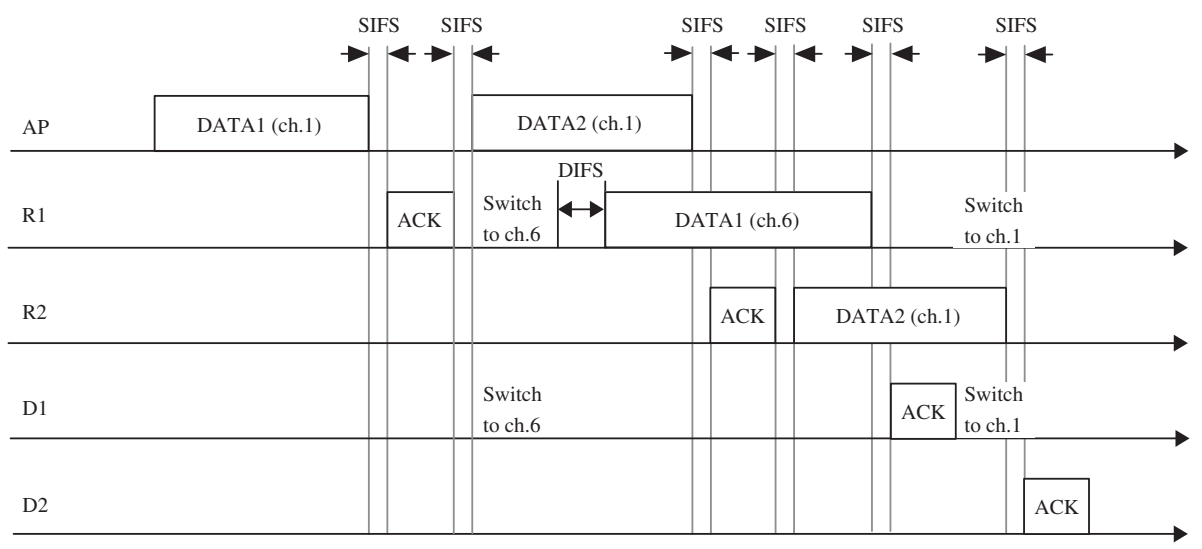

Figure 7. The timing diagram of DATA transmission in a multiple-frame relay mode. ACK, acknowledgement; AP, access point; DIFS, distributed coordination function interframe space; SIFS, short interframe space.

R2 continuously. After receiving DATA2, R2 sends ACK to AP as a response. Then, R2 forwards it to D2 using the primary channel (i.e., channel 1) and D2 sends ACK to R2 as a response after receiving DATA2. Meanwhile, R1 and D1 switch to channel 6 immediately after R1 transmits ACK. When channel 6 is idle during $t D I F S, \mathrm{R} 1$ starts to transmit DATA1 to D1 without backoff. When D1 receives DATA1 successfully, it replies with ACK. After DATA1 and ACK are exchanged between $\mathrm{R} 1$ and D1, they return to the primary channel (i.e., channel 1) and wait to participate in the next channel contention.

Now, we describe the MRMAC operation in exceptional cases such as when either receivers or relay nodes fail to transmit frames because of interference, channel error, or busy channel status. The interference may occur because of the transmission of hidden nodes, whereas channel error may occur by either node mobility or channel condition variation. And a relay node may not attempt to transmit a data frame to its intended receiver because of the busy secondary channel. Under this situation, MRMAC operates as follows. First, when AP fails to transmit a frame to a relay node, both the relay node and the corresponding receiver do not switch to the allocated channel and wait for the next channel contention. The receiver can know that the relay node failed to receive a data frame because it cannot overhear ACK from its relay node. Likewise, AP can know that the relay node did not receive a data frame from the absence of ACK. Once detecting a frame loss and being ready for the next frame transmission, AP will try to send the next frame in $\hat{s}$.

Second, when a relay node fails to send a data frame, it takes responsibility for retransmitting the frame. Relay nodes follow the DCF operation when trying to transmit the failed frames. In a bad channel condition, when a relay node fails to receive an ACK frame from the receiver, it returns to the primary channel after the time required for ACK reception, whereas a receiver returns to the channel after the time required for receiving the data frame. And, in the busy channel, once sensing the secondary channel as busy, it returns to the primary channel immediately without the backoff procedure and then will wait for the next channel access. The intended receiver returns to the primary channel after the intended time for the data frame reception. Thus, they can participate to the next channel access at the primary channel in time in both conditions. However, when relay nodes send those failed frames to the intended receiver, if they have their own uplink frames toward the AP, the fairness problem among the uplink nodes may occur. The reason is that relay nodes might use the channel access opportunity not for their own uplink frames but for the other frames to be relayed. For handling this problem, they use back-to-back transmissions for sending multiple frames. When a relay node seizes the medium after contention, it transmits one uplink frame and all the other frames, which should be relayed to the intended receivers. By doing so, fairness among uplink nodes could be guaranteed.

In an uplink transmission, when a node transmits data frames to AP, it looks up its staRelayTable to find a relay node. If one or more relay nodes exist, a node transmits its frame via the relay node, 
which is similar to the operation of a single-frame relay mode in Figure 6. First, a node exchanges GRTS and CTS with AP. Then, it sends a data frame to the selected relay node. After responding to the node, the relay node forwards the received frame to AP. If there is no relay node, the node will send the frame to AP directly.

\subsection{Effect of switching delay}

In the multiple-frame relay mode of MRMAC, a receiver and relay node switch to a secondary channel, exchange a data frame between them, and then return to the primary channel. Because these nodes have to switch channels twice per frame, someone may think that the switching delay, the time elapsed for switching channels, could degrade the network performance.

However, the impact of switching delay on network performance is negligible. We explain it with an example shown in Figure 7. In the example, we assume that the length of both DATA1 and DATA2 is $L$. In the figure, the operation of DATA1 transmission between R1 and D1 occurs concurrently with the operation of DATA2 transmission among AP, R2, and D2 using different channels. The required time for exchanging a data frame between R1 and D1 can be calculated as $T 1=2 \delta+t D I F S+T\left(L, R_{\mathrm{R} 1, \mathrm{D} 1}\right)+t$ SIFS $+T_{\mathrm{ACK}}$, where $\delta$ is the switching delay. Also, we can calculate that the required time for exchanging a data frame between AP and D2 is $T 2=T_{\text {MRMAC }}(L, R 2, D 2)$ from Equation (2). If R1 and D1, which use the secondary channel, return to the primary channel before the end of DATA2 transmission between R2 and D2 (i.e., $T 1 \leqslant T 2$ ), the impact of the switching delay on network performance can be ignored. From the decision algorithm, we know that the frame with the largest $T\left(L, R_{\mathrm{R}, \mathrm{D}}\right)$ value is served last, and thus $T\left(L, R_{\mathrm{R} 2, \mathrm{D} 2}\right)$ is greater than or equal to $T\left(L, R_{\mathrm{R} 1, \mathrm{D} 1}\right)$. Let us consider the worst case where $T\left(L, R_{\mathrm{R} 2, \mathrm{D} 2}\right)$ is equal to $T\left(L, R_{\mathrm{R} 1, \mathrm{D} 1}\right)$. In this case, $T 1 \leqslant T 2$ can be expressed as $2 \delta \leqslant T\left(L, R_{\mathrm{AP}, \mathrm{R} 2}\right)+2 t S I F S+T_{\mathrm{ACK}}-t D I F S$. Then, we can derive it as $\delta \leqslant T_{\mathrm{ACK}}$ approximately because $T\left(L, R_{\mathrm{AP}, \mathrm{R} 2}\right)$ is greater than $\left(T_{\mathrm{ACK}}+3 t\right.$ SIFS $)$ always. Thus, if $\delta$ is smaller than or equal to $T_{\mathrm{ACK}}$, the performance degradation by $\delta$ does not occur because $T 1$ is smaller than $T 2$ always in the condition. For example, $\delta$ in off-the-shelf $802.11 \mathrm{~b}$, hardware is about 150 to $200 \mu \mathrm{s}$ [26], but $T_{\mathrm{ACK}}$ is about $300 \mu \mathrm{s}$.

\subsection{Throughput analysis}

In this section, we derive the maximum throughput of MRMAC in a given network where there exist $m$ available channels (i.e., one primary channel and $(m-1)$ secondary channels) and several downlink flows of $L$ byte size with average $R \mathrm{Mb} / \mathrm{s}$. For simplicity, we assume that there are a sufficient number of relay nodes (named as R1, R2, and so on) so that $m$ back-to-back transmissions always could be initiated. Also, we assume the ideal channel condition (i.e., no hidden terminals), no uplink flows (i.e., no collisions), and saturated traffic for each flow. Last, we ignore the processing delay of the decision algorithm.

In these circumstances, because $m$ frames are transmitted in a single-channel access in MRMAC, the MRMAC's expected throughput can be estimated as

$$
E T_{\mathrm{MRMAC}}=\frac{m \cdot L \cdot 8}{t D I F S+t \text { Backoff }+T_{\mathrm{MRMAC}}(m)}
$$

where tBackoff is the average time spent for backoff (i.e., $C W_{\min } / 2 \cdot t$ SlotTime in which $C W_{\min }$ is the value for the minimum contention window) and $T_{\text {MRMAC }}(m)$ is the total time required for sending $m$ data frames. We can calculate $T_{\text {MRMAC }}(m)$ as $T_{\text {GRTS }}(m)+m \cdot\left(t \operatorname{SIFS}+T_{\mathrm{CTS}}\right)+$ $\sum_{i=1}^{m}\left(t S I F S+T\left(L, R_{\mathrm{AP}, \mathrm{Ri}}\right)+t\right.$ SIFS $\left.+T_{\mathrm{ACK}}\right)+t S I F S+T\left(L, R_{\mathrm{R}, \mathrm{D}}^{\prime}\right)+t S I F S+T_{\mathrm{ACK}}$, where $T_{\mathrm{GRTS}}(m)$ is the transmission time for GRTS with information about $m$ frames and $R_{\mathrm{R}, \mathrm{D}}^{\prime}$ is the minimum transmission rate of $m$ transmission rates between selected relay nodes and receivers. If $R_{\mathrm{r} 1}$ is the average transmission rate between $\mathrm{AP}$ and selected relay nodes and $R_{\mathrm{r} 2}$ is the average rate of the minimum transmission rates (i.e., $\left.R_{\mathrm{R}, \mathrm{D}}^{\prime}\right), T_{\mathrm{MRMAC}}(m)$ could be expressed as 


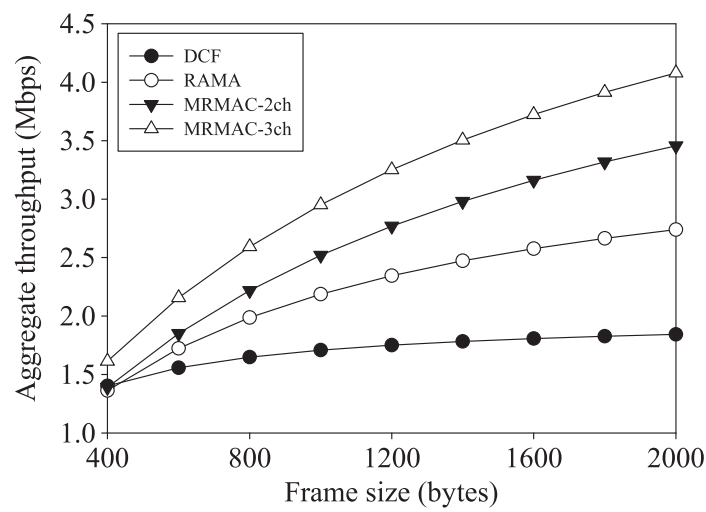

Figure 8. Throughput analysis of multichannel relay medium access control (MRMAC), relay-aided media access (RAMA), distributed coordination function (DCF) with two-way handshake according to the frame size.

$T_{\mathrm{GRTS}}(m)+m \cdot T_{\mathrm{CTS}}+m \cdot T\left(L, R_{\mathrm{r} 1}\right)+T\left(L, R_{\mathrm{r} 2}\right)+(m+1) \cdot T_{\mathrm{ACK}}+(3 m+2) \cdot t$ SIFS. In the same manner, the expected throughputs of both RAMA and DCF with two-way handshake could be estimated easily.

Figure 8 shows the results of throughput analysis for MRMAC, RAMA, and DCF according to data sizes ranging from 400 to 2000 bytes. We set $C W_{\min }$ at $31, R$ at $2 \mathrm{Mb} / \mathrm{s}, R_{\mathrm{r} 1}$ at $11 \mathrm{Mb} / \mathrm{s}$, and $R_{r 2}$ at $5.5 \mathrm{Mb} / \mathrm{s}$. Also, $m$ is set to either 2 or 3 . In the figure, 'MRMAC-2ch' denotes MRMAC protocol with two channels and 'MRMAC-3ch' denotes MRMAC protocol with three channels. The results in the data size of lower than 400 bytes are not indicated in the figure because no gain is expected by MRMAC and RAMA over DCF. Except in this case, we can see that MRMAC shows improved performance over RAMA and DCF. Specifically, when the data size increases, MRMAC shows much better improvement than other protocols. For example, when the data size is 600 bytes (or 2000 bytes), the aggregate throughput of MRMAC-2ch and MRMAC-3ch is about $7.4 \%$ and $25.1 \%$ (26.1\% and $48.9 \%$ ) better than RAMA, respectively.

\subsection{Discussion}

Now, we examine some issues that should be considered for incorporating MRMAC into IEEE 802.11. First, AP should implement the decision algorithm that selects multiple frames to be served in a single-channel access. Second, the modifications in some frames (e.g., GRTS, ACK, and DATA) are required. GRTS, a new control frame, should be defined for initiating the medium access operation for MRMAC where the frame format is shown in Figure 4. And ACK needs to be modified to include the MAC address of a transmitter. Also, when a transmitter sends DATA to be relayed to the corresponding relay node, it should include the MAC address of the intended receiver in the DATA. For this, a transmitter exploits the reserved address 4 field in the MAC header. Last, nodes should modify DCF operations so that they follow the MRMAC's medium access operation.

Although these modifications make it difficult to adopt MRMAC in IEEE 802.11 standards, these are not just a matter of MRMAC. Most of the relay-based MAC protocols [15-17] are also required to change the operation in IEEE 802.11 standards as well as to define some control frames to enable the relay operation. Nevertheless, MRMAC has better throughput and delay performance than the other relay-based MAC protocols [15-17]. Now, we explain whether MRMAC can be integrated with the practical relay MAC protocol such as [18] or not. Lim et al. [18] have proposed a practical relay MAC protocol using proxy nodes that act as a relay node. The approach does not need the modification of nodes, which is its main advantage when compared with the previous approaches [15-17], and thus is easy to deploy. However, because it requires new nodes (i.e., proxy node) supporting relay capability, it is not cost-effective. Unfortunately, MRMAC cannot adapt the approach because the nodes should obtain some information from GRTS such as channel information to change its channel to a secondary channel. 


\section{PERFORMANCE EVALUATION}

In this section, we evaluate the performance of MRMAC by using an ns-2 simulator [19] and compare it with DCF without RTS/CTS and RAMA [16], one of the representative frame-relaying protocols. We assume that nodes can select the optimal rate according to the current channel condition in DCF. Also, RAMA uses RTS and CTS exchange only when the relay transmission between a transmitter and a receiver is enabled. In the direct transmission, RAMA follows DCF to reduce control overhead. And we do not compare MRMAC with SI-CCMAC [14] because SI-CCMAC has difficulty obtaining an interference map in the network in mobile environments. In our simulations, the two-ray path loss model is used as the radio propagation model and the random-way point mobility model is used as the mobility model [27]. We set the nodes such that each has an IEEE $802.11 \mathrm{~b}$ interface with IEEE $802.11 \mathrm{~b}$ parameters shown in Table II. AP can use three nonoverlapping channels that are not used by nearby APs; the queue size of AP is set to 100. The transmission power of nodes is set to $0.001 \mathrm{~W}$ (i.e., $0 \mathrm{dBm}$ ), where the transmission range of the base rate (i.e., $1 \mathrm{Mb} / \mathrm{s}$ ) with the power is about $340 \mathrm{~m}$. Also, each relay candidate node sends advertisement messages to AP every $2 \mathrm{~s}$.

In our simulation, we used both User Datagram Protocol (UDP) and Transfer Control Protocol (TCP) flows, and all nodes except AP become receivers of these flows. In UDP, AP generates constant-bit-rate traffic per node. We measured the performance of DCF, RAMA, and MRMAC by varying the number of nodes and the traffic rate. In TCP, TCP NewReno is used, and the TCP frame size is set to 1024 bytes. We measured the TCP performance of the three protocols by varying the number of nodes. We measured aggregate throughput and delay in the case of UDP and aggregate throughput and round-trip time (RTT) in the case of TCP. The aggregate throughput is the total sum of DATA delivered to receivers per second during the simulation time. The delay is defined as the time difference between the time when a frame is inserted to the queue of a transmitter and the time when the frame is delivered successfully to the intended receiver. RTT is defined as the time difference between the time when the source node sends a TCP frame and the time when it receives the ACK of the frame.

\subsection{User Datagram Protocol Performance according to the number of nodes}

We observed the impact of the number of relay nodes on the UDP performance by varying the number of nodes except AP from 10 to 40. AP is in the center of a circle whose radius is $340 \mathrm{~m}$, and all nodes move at a maximum speed of $5 \mathrm{~m} / \mathrm{s}$ within the circle. AP generates UDP traffic with a constant bit rate of $1 \mathrm{Mb} / \mathrm{s}$ where the network is saturated and the number of downlink flows is

Table II. Simulation parameters

\begin{tabular}{lc}
\hline Parameters & Values \\
\hline Preamble length & $144 \mathrm{bits}$ \\
PLCP header length & $48 \mathrm{bits}$ \\
MAC header & $272 \mathrm{bits}$ \\
SlotTime & $20 \mu \mathrm{s}$ \\
Short interframe space & $10 \mu \mathrm{s}$ \\
DCF interframe space & $50 \mu \mathrm{s}$ \\
Request to send & $352 \mu \mathrm{s}$ \\
Clear to send & $304 \mu \mathrm{s}$ \\
ACK in DCF and relay MAC/ACK in MRMAC & $304 \mu \mathrm{s} / 352 \mu \mathrm{s}$ \\
$C W_{\text {min }}$ & 31 \\
$C W_{\text {max }}$ & 1023 \\
Data rates & $1,2,5.5$, and $11 \mathrm{Mb} / \mathrm{s}$ \\
Channel switching delay & $224 \mu \mathrm{s}$ \\
\hline
\end{tabular}

ACK, acknowledgement; DCF, distributed coordination function; MAC, medium access control; MRMAC, multichannel relay MAC. 


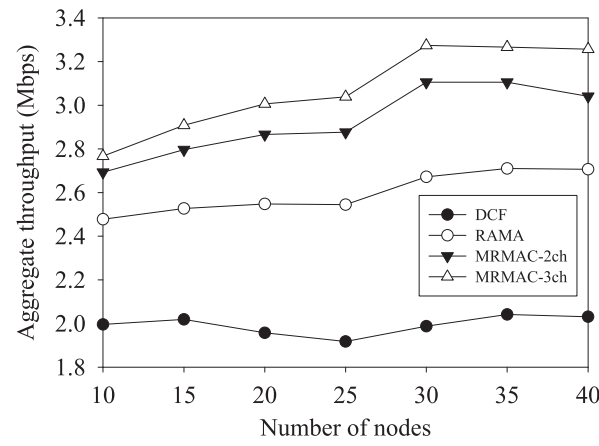

(a)

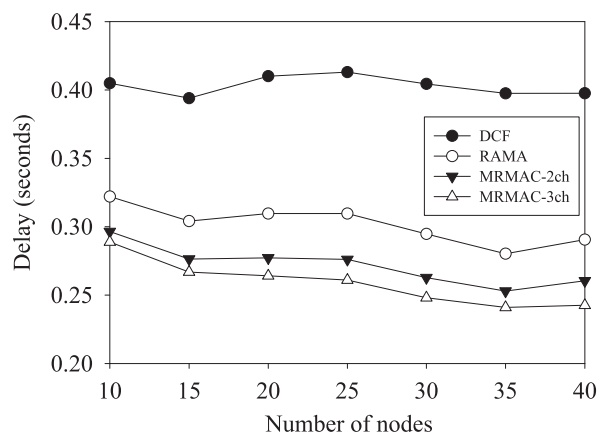

(b)

Figure 9. Comparisons of User Datagram Protocol performance with varying numbers of nodes. (a) Aggregate throughput. (b) Delay. DCF, distributed coordination function; RAMA, relay-aided media access; MRMAC, multichannel relay medium access control.

set to 10 . The simulation time per scenario is $300 \mathrm{~s}$, and the frame size is set to 1024 bytes. Twenty random topologies are generated per a certain number of nodes, and a dot in Figure 9 is the average value for the 20 simulations.

Figure 9 illustrates the UDP performance of DCF, RAMA, and MRMAC as a function of the number of nodes. From the figure, we can see that MRMAC shows better performance than DCF and RAMA. The performance improvement of MRMAC over the other protocols increases gradually as the number of nodes increases. For example, when the number of nodes is 10 , the UDP aggregate throughput (or delay) achieved by MRMAC-2ch is about $9.1 \%$ and $35.4 \%$ (8.5\% and $27.1 \%$ ) better than RAMA and DCF, respectively. When the number of nodes is 25 , the throughput improvement (or delay) of MRMAC-2ch over RAMA and DCF is $13.0 \%$ and $47.7 \%$ $(10.8 \%$ and $33.1 \%)$, respectively. Also, we can see that MRMAC-3ch shows better performance than MRMAC-2ch.

The reason for these results is as follows. Generally, as the number of nodes in the network increases, the number of possible relay nodes also increases. Thus, because the number of relaypossible frames, which can be delivered to receivers via relay nodes increases, RAMA and MRMAC outperform DCF. Because MRMAC enables concurrent transmissions for those relay-possible frames, the time required to transmit the same number of relay-possible frames in MRMAC is reduced compared with that required in RAMA, and thus RAMA shows worse performance than MRMAC. Last, because the degree of concurrency is proportional to the number of available channels, the performance of MRMAC-3ch is higher than that of MRMAC-2ch.

\subsection{User Datagram Protocol performance according to the flow rates}

In this subsection, we compare the performance of MRMAC with that of DCF and RAMA by varying the traffic rate per flow from 40 to $240 \mathrm{~kb} / \mathrm{s}$, where the number of nodes except AP is 20 and the number of receivers is 20 where each receiver has one flow. The other simulation settings such as AP position, node movement, simulation time, and the frame size are the same as previous simulations.

Figure 10 shows the aggregate throughput and delay as a function of traffic rate per flow. When the traffic rate per flow is larger than a certain rate, MRMAC outperforms both DCF and RAMA. For example, when the traffic rate is $160 \mathrm{~kb} / \mathrm{s}$, the throughput improvement (or delay) of MRMAC-2ch over RAMA and DCF is $11.9 \%$ and $32.5 \%$ (18.9\% and 36.3\%), respectively. The performance gain of MRMAC-3ch is higher than MRMAC-2ch. This is because concurrent transmissions of multiple frames by MRMAC reduce the time elapsed for transmitting frames. Also, in MRMAC, the time can further be reduced as the number of nonoverlapping channels increases because the degree of concurrency increases. 


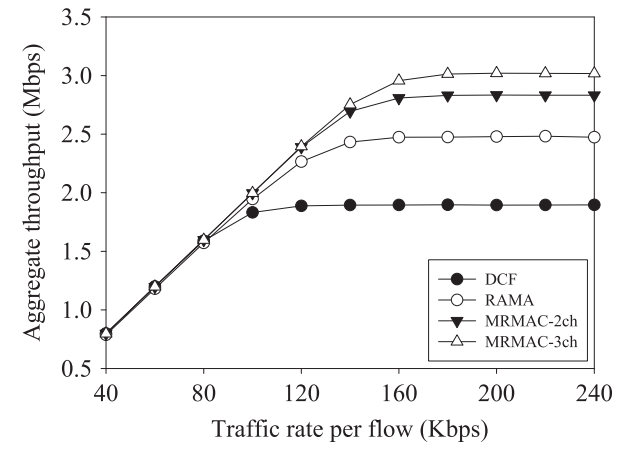

(a)

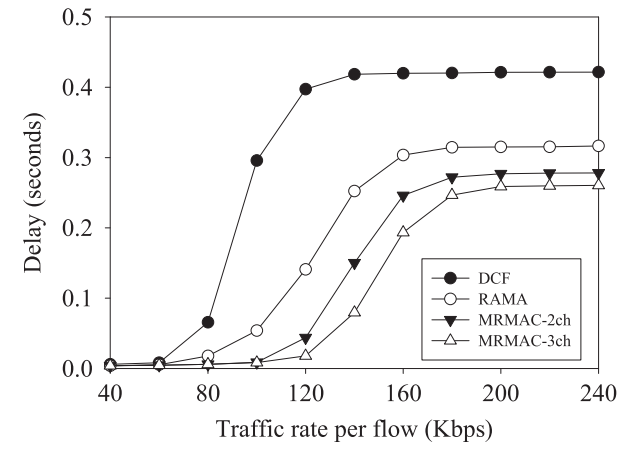

(b)

Figure 10. Comparisons of User Datagram Protocol performance with varying traffic rates per flow. (a) Aggregate throughput. (b) Delay. DCF, distributed coordination function; RAMA, relay-aided media access; MRMAC, multichannel relay medium access control.

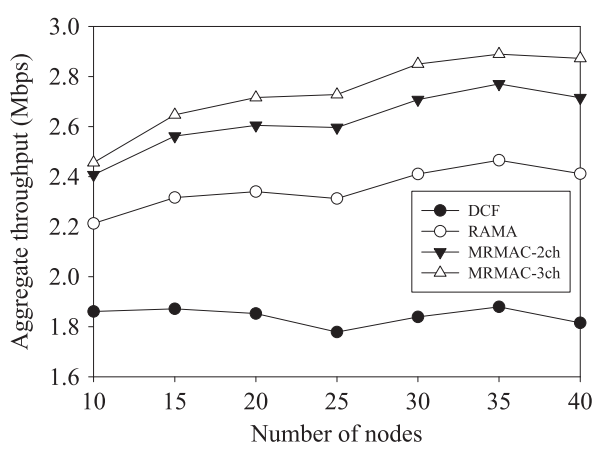

(a)

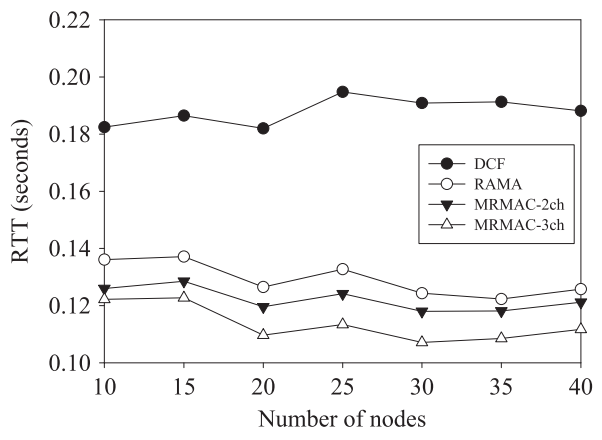

(b)

Figure 11. Comparisons of Transfer Control Protocol performance with varying numbers of nodes. (a) Aggregate throughput. (b) Round-trip time. DCF, distributed coordination function; RAMA, relay-aided media access; MRMAC, multichannel relay medium access control.

\subsection{Transfer Control Protocol performance according to the number of nodes}

Now, we evaluate the TCP performance of MRMAC according to the number of nodes to observe the impact of the number of relay nodes. Simulation environments are the same as that in Section 5.1. Figure 11 shows the TCP performance of DCF, RAMA, and MRMAC. Similar to the simulation results in Section 5.1, we can identify that MRMAC shows better throughput and RTT performance than the other protocols for the same reason described in the previous subsections. For example, when the number of nodes is 20, the TCP aggregate throughput (or RTT) achieved by MRMAC-2ch is about $11.3 \%$ and $40.6 \%$ (5.5\% and $34.2 \%$ ) better than RAMA and DCF, respectively. Also, we can see that the performance gain of MRMAC-3ch is higher than that of MRMAC-2ch. For example, when there are 25 nodes, the TCP aggregate throughput (or RTT) by MRMAC- $3 \mathrm{ch}$ is about $5.1 \%$ $(8.7 \%)$ better than MRMAC-2ch.

\subsection{User Datagram Protocol performance according to the number of contending nodes}

Last, we look at how the UDP performance of three protocols changes as the contention level increases. For this, we vary the number of uplink nodes from 0 to 10 with a $200-\mathrm{kb} / \mathrm{s}$ traffic rate for each uplink flow where the number of nodes except AP is 30. The uplink node is randomly selected. The other simulation environments are the same as that in Section 5.1. 


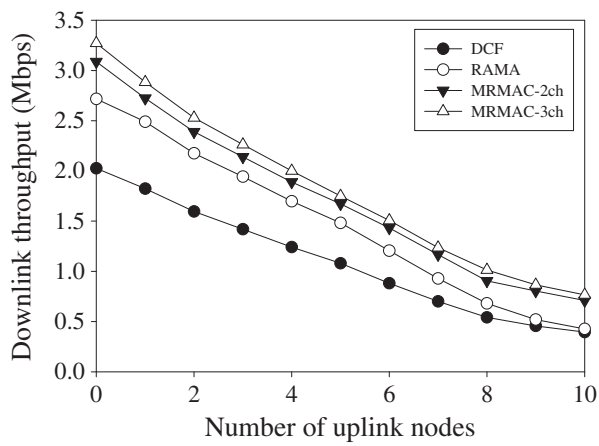

(a)

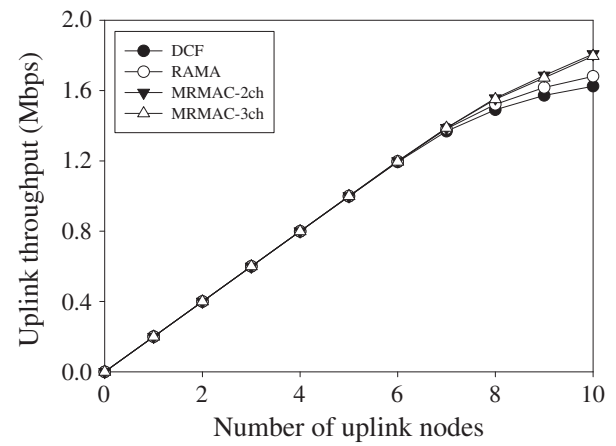

(b)

Figure 12. Comparisons of User Datagram Protocol performance with varying numbers of uplink nodes. (a) Downlink throughput. (b) Uplink throughput. DCF, distributed coordination function; RAMA, relayaided media access; MRMAC, multichannel relay medium access control.

As shown in Figure 12, when the number of uplink nodes increases, the downlink throughput of three protocols gets worse because of the increased contention level but that of MRMAC still outperforms that of the others, similar to the previous simulation results. Furthermore, the more the contention level imposes, the more is the downlink throughput gain in terms of the ratio of MRMAC to the others. For example, when the number of uplink nodes is two, MRMAC-2ch provides a higher downlink throughput than DCF and RAMA at about $49.7 \%$ and $9.8 \%$, respectively. But, when the number of uplink nodes is 10, the downlink throughput achieved by MRMAC-2ch is better than DCF and RAMA at about $79.3 \%$ and $66.2 \%$, respectively. Also, we can see that the uplink throughput in MRMAC is higher than that of the others specially in the high-contention environments. MRMAC can provide a higher downlink throughput than the others with the help of the concurrent transmissions of multiple downlink frames. Also, more time can be used for the uplink traffic with the same reason, and thus, the uplink throughput of MRMAC is higher than that of the others.

\section{CONCLUSION}

In this paper, we proposed an enhanced MAC protocol called MRMAC that enhances the network throughput and delay of downlink transmissions in WLANs. The proposed MAC protocol has been designed to enable interference-free concurrent transmissions by utilizing frame-relaying approaches and several nonoverlapping channels in IEEE 802.11. MRMAC exploits frame-relaying transmissions via relay nodes to allow transmission concurrency and multiple nonoverlapping channels to enable interference-free transmissions among links. We designed a decision algorithm, which consists of a frame selection algorithm to select frames to be transmitted and relay nodes to participate in the transmissions and a channel assignment algorithm to assign different channels for those frames. In addition, we proposed a medium access operation for concurrent data frame transmissions. Also, we explain the effect of switching delay, execute throughput analysis, and discuss some issues considered for incorporating MRMAC into IEEE 802.11. We showed the effectiveness of the proposed protocols via extensive simulation results.

\section{ACKNOWLEDGEMENTS}

This research was supported by a World Class University program funded by the Ministry of Education, Science, and Technology through the National Research Foundation of Korea (R31-10100). This research was supported by the MKE (The Ministry of Knowledge Economy), Korea, under the ITRC 
(Information Technology Research Center) support program supervised by the NIPA (National IT Industry Promotion Agency) (NIPA-2012-H0301-12-3002). This work was supported by a National Research Foundation of Korea (NRF) grant funded by the Korean government (MEST) (2011-0029034).

\section{REFERENCES}

1. IEEE 802.11-2007. IEEE Standard—part 11: wireless LAN medium access control and physical layer specifications, June 2007.

2. Yang X, Vaidya NH. On the physical carrier sense in wireless ad hoc networks. Proceedings of IEEE INFOCOM, Miami, FL, USA, March 2005; 2525-2535.

3. Zhu J, Guo X, Yang LL, Conner WS. Leveraging spatial reuse in 802.11 mesh networks with enhanced physical carrier sensing. Proceedings of IEEE ICC, Paris, France, June 2004; 4004-4011.

4. Muqattash A, Krunz M. Power controlled dual channel (PCDC) medium access protocol for wireless ad hoc networks. Proceedings of IEEE INFOCOM, San Francisco, California, USA, March 2003; 470-480.

5. Muqattash A, Krunz M. A single-channel solution for transmission power control in wireless ad hoc networks. Proceedings of ACM MobiHoc, Tokyo, Japan, May 2004; 210-221.

6. Monks JP, Bharghavan V, Mei W, Hwu W. A power controlled multiple access protocol for wireless packet networks. Proceedings of IEEE INFOCOM, Anchorage, Alaska, USA, April 2001; 219-228.

7. Akella A, Judd G, Steenkiste P, Seshan S. Self management in chaotic wireless deployments. Proceedings of ACM MobiCom, Cologne, Germany, August 2005; 185-199.

8. Kim TS, Lim H, Hou JC. Understanding and improving the spatial reuse in multihop wireless networks. IEEE Transactions on Mobile Computing 2008; 7(10):1200-1212.

9. Wang JT. Joint rate regulation and power control for cochannel interference limited wireless networks. Wiley International Journal of Communication Systems 2011; 24(8):967-977.

10. So J, Vaidya N. Multi-channel MAC for ad hoc networks, handling multi-channel hidden terminals using a single transceiver. Proceedings of ACM MobiHoc, Tokyo, Japan, May 2004; 222-233.

11. Bahl P, Chandra R, Dunagan J. SSCH: slotted seeded channel hopping for capacity improvement in IEEE 802.11 ad-hoc wireless networks. Proceedings of ACM MobiCom, Philadelphia, PA, USA, September 2004; 216-230.

12. Kao HH, Wu PJ, Lee CN. Analysis and enhancement of multi-channel MAC protocol for ad hoc networks. Wiley International Journal of Communication Systems 2011; 24(3):310-324.

13. Hu Z, Tham C-K. CCMAC: coordinated cooperative MAC for wireless LANs. Proceedings of ACM MSWiM, Vancouver, Canada, October 2008; 60-69.

14. Hu Z, Tham C-K. SI-CCMAC: sender initiating concurrent cooperative MAC for wireless LANs. Proceedings of ACM WiOpt, Seoul, Korea, June 2009; 1-10.

15. Zhu H, Coa G. rDCF: a relay-enabled medium access control protocol for wireless ad hoc networks. IEEE Transactions on Mobile Computing 2006; 5(9):1201-1214.

16. Zou S-H, Li B, Wu H-T, Zhang Q, Zhu W-W. A relay aided media access (RAMA) protocol in multi-rate wireless networks. IEEE Transactions on Vehicular Technology 2006; 55(5):1657-1667.

17. Liu P, Tao Z, Narayanan S, Korakis T, Panwar SS. CoopMAC: a cooperative MAC for wireless LANs. IEEE Journal on Selected Areas in Communications 2007; 25(2):340-354.

18. Lim WS, Kim DW, Suh YJ. PR-MAC: a practical approach for exploiting relay transmissions in multi-rate WLANs. IEEE Transactions on Wireless Communications 2010; 9(1):66-71.

19. NS Official Website. (Available from: http://www.isi.edu/nsnam/ns/).

20. Liu W, Jin H, Wang X, Guizani M. A novel IEEE 802.11-based MAC protocol supporting cooperative communications. Wiley International Journal of Communication Systems 2011; 24(11):1480-1495.

21. An D, Woo H, Yoon H, Yeom I. Enhanced cooperative communication MAC for mobile wireless networks. Elsevier Computer Networks 2013; 57(1):99-116.

22. Rui X, Hou J, Zhou L. Decode-and-forward with full-duplex relaying. Wiley International Journal of Communication Systems 2012; 25(2):270-275.

23. Xu W, Lin J, Niu K, He Z. Error performance for relaying protocols with multiple decode-and-forward relays. Wiley International Journal of Communication Systems 2010; 23(8):1016-1040.

24. Pavon JP, Choi S. Link adaptation strategy for IEEE 802.11 WLAN via received signal strength measurement. Proceedings of IEEE ICC, Anchorage, Alaska, USA, May 2003; 1108-1113.

25. IEEE 802.11e. Part 11: wireless medium access control (MAC) and physical layer (PHY) specifications: medium access control (MAC) enhancements for quality of service (QoS), supplement to IEEE 802.11 standard, November 2005.

26. Maxim Integrated Products Inc. Sunnyvale, California, USA MAX2820, MAX2820A,MAX2821, MAX2821A 2.4GHz 802.11b Zero-IF Transceivers Data Sheet Rev. 5; May 2005.

27. Rappaport T. Wireless Communications: Principle and Practice. Prentice Hall: Upper Saddle River, NJ, 2001. 


\section{AUTHORS’ BIOGRAPHIES}

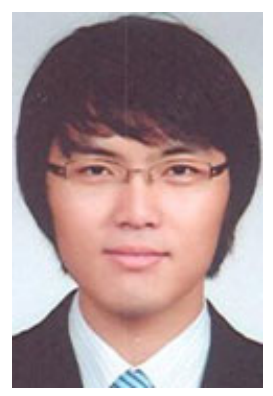

Dong-Wook Kim received his BS degree in Computer Engineering from Kyungpook National University, Daegu, Korea, in 2005 and his $\mathrm{PhD}$ degree in Computer Science and Engineering at Pohang University of Science and Technology (POSTECH), Pohang, Korea, in 2012. He is now with the Smart Grid Security Research Department, the Attached Institute of ETRI, Daejeon, Korea. His research interests include wireless LAN MAC protocol, mobility management, wireless network security, and smart grid security.

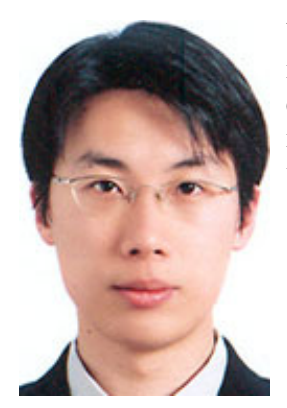

Wan-Seon Lim received the $\mathrm{BS}, \mathrm{MS}$, and $\mathrm{PhD}$ degrees in computer science and engineering at Pohang University of Science and Technology (POSTECH), Pohang, Korea. He is currently a research fellow in the University of Michigan, Ann Arbor, MI. His research interests include wireless LAN MAC protocol, ad hoc networks, and video streaming over wireless networks.

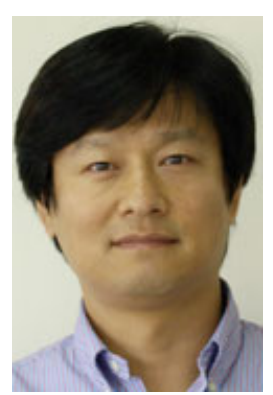

Young-Joo Suh received his BS and MS degrees in Electronics Engineering from Hanyang University, Seoul, Korea, in 1985 and 1987, respectively, and his PhD degree in Electrical and Computer Engineering from Georgia Institute of Technology, Atlanta, Georgia, in 1996. He is currently a professor in the Department of Computer Science and Engineering at the Pohang University of Science and Technology (POSTECH), Pohang, Korea. From 1988 to 1990 , he was a research engineer at the Central Research Center of LG Electronics Inc., Seoul, Korea. From 1990 to 1993, he was an assistant professor in the Department of Computer Science and Engineering at Chung-Cheong College, Korea. After receiving his $\mathrm{PhD}$, he worked as a postdoctoral researcher in the Computer Systems Research Laboratory in the School of Electrical and Computer Engineering at the Georgia Institute of Technology from 1996 to 1997. From 1997 to 1998 , he was a research fellow of the Real-Time Computing Laboratory in the Department of Electrical Engineering and Computer Science at the University of Michigan, Ann Arbor, MI. His current research interests include wireless LAN MAC protocol, mobility management, ad hoc networks, 4G wireless mobile networks, and so on. Dr. Suh is a member of the IEEE and the IEEE Communications Society. 